\title{
On improving the quality of VoIP connections
}

\author{
A A Bukatov ${ }^{1}$, D Y Polukarov ${ }^{2}$, N D Zaitsev ${ }^{3}$ and A M Sukhov ${ }^{2}$ \\ ${ }^{1}$ Southern Federal University, Stachki str. 200/1, k.213, Rostov-on-Don, Russia, 344090 \\ ${ }^{2}$ Samara National Research University, Moskovskoe Shosse 34A, Samara, Russia, 443086 \\ ${ }^{3}$ Don State Technical University, Lenina str. 69, Rostov-on-Don, Russia, 344079
}

\begin{abstract}
Improving the quality of VoIP connections is a very important goal in the area of telecommunications. The proportion of multimedia traffic in relation to the total traffic supported by providers is constantly increasing. To identify and troubleshoot issues with VoIP connections, network providers need both criteria and a methodology for assessing connection quality. We offer a methodology for assessing the quality of VoIP connections. A comparative analysis of VoIP codecs is also made.
\end{abstract}

\section{Introduction}

The proportion of multimedia traffic as compared to the total traffic supported by providers is constantly increasing [1]. Hence, much attention is paid to the quality of VoIP services. There is a need to define criteria for assessing the quality of VoIP connections in order to improve the quality of telecommunications services.

The existing methods for assessing speech quality across VoIP connections are analyzed in [2]. The authors of [2] chose what they considered to be the best audio codec in this context.

The following types of voice signal distortion are considered in [2]:

- voice stream delay;

- intermittent and illegible speech;

- extraneous noise;

- echoes;

- unnatural voice (robotic voice effects);

- abnormal (too low) signal volume.

Further, in [3], four characteristics of network connections are considered: i.e., bandwidth, delay, loss, and the variation of the delay (jitter). All these characteristics are measured in terms of three grades: Good, Acceptable, Poor. Thus, the compound metric is comprised of four values which each take one of the grades G-A-P (Good, Acceptable, or Poor).

This approach is more general and formal than others which have been proposed, and therefore more universal and suitable for automation.

However, the approach implemented in [3] is specifically for audiovisual traffic (VVoIP connections). We see the advisability of using this approach for VoIP codecs, as well, discussed in [2].

\section{Related work}

Objective methods for assessing the quality of a voice signal are widely studied in $[4,5,6,7,8]$. In these works, various methods are offered: Emodel, PSQM / PSQM +, PESQ, P.563. These will be described in more detail below. 
In [2] methods are proposed for constructing tools for monitoring the quality of voice flow transmissions. The development of these tools were based on the use of VoIP telephony systems to support the activities of educational institutions using distance learning forms to enhance the distance learning process.

In [3] a new mechanism for providing online assessments of VVoIP quality of service is introduced. This operates on network paths without the participation of users. The mechanism uses the "GAP-model", which is a model for measuring the QoE in terms of measurable network factors such as bandwidth, delay, jitter and loss (see above).

\section{Overview of assessment methods}

Speech quality assessment methods for VoIP systems are subdivided into subjective and objective metrics [9]. Subjective methods require that an expert evaluates the situation in question, and therefore are unacceptable in relation to automatic evaluation. Objective methods for assessing the quality of voice transmission are divided into two groups. The first group assesses the quality of transmission of primary data streams. This group includes Emodel [4]. The second group evaluates the quality of the audio stream transmission itself. The second group includes the PSQM/PSQM+ method (Perceptual Speech Quality Measure) [5], which has been further developed into the PESQ method (Perceptual Evaluation of Speech Quality) [6]; this second group also includes the method P.563 [7, 8].

The results of a comparative analysis of voice quality estimation methods for VoIP systems are given in Table 1 [2].

Table 1.Comparison of objective speech quality analysis methods.

\begin{tabular}{lccc}
\hline & Emodel & PESQ & P.563 \\
\hline The absence of excess traffic & yes & no & yes \\
\hline $\begin{array}{l}\text { The possibility of one-way data flow analysis } \\
\begin{array}{l}\text { The possibility of analyze the types of distortion of the } \\
\text { received speech stream }\end{array}\end{array}$ & no & no & yes \\
\hline
\end{tabular}

\section{Comparing codecs and choosing the best one}

There are network routes that are comparatively long but connect, end-to-end, quite closely spaced nodes.

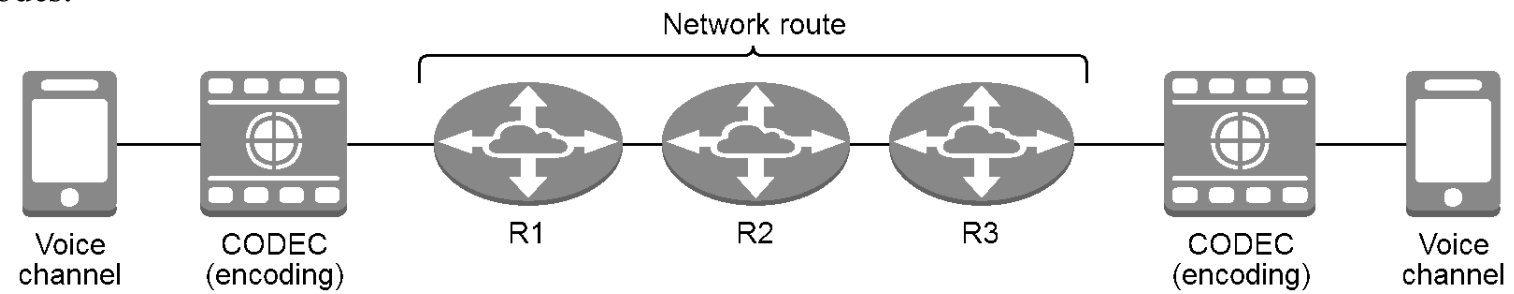

Figure 1. Scheme of transmission voice signal between VoIP-phones.

For example, consider these cases.

Case 1: a subscriber VoIP phone is connected to the corporate telecommunications network of the Southern Federal University (SFU) through the LTE (mobile 4G network) of the mobile operator MTS. The VoIP subscriber is located in the city of Rostov-on-Don.

The route shown in Figure 2 can be described by the following sequence of cities: Rostov-on-Don Moscow - St. Petersburg - Helsinki - Stockholm - Amsterdam - St. Petersburg - Moscow - Rostov-onDon. It should be noted that the shorter return route from Amsterdam to St. Petersburg (which reduces total length of the route) is due to the highly developed infrastructure of the international channels of the branch network of the Ministry of Education and Science of the Russian Federation RUNNet [10].

It is known that the transmission of information flows through "long" routes subjects such streams to certain kinds of distortion [11], such as those caused by delays in packet delivery (tempo distortions) and those caused by packet loss - in the case of data transfer protocols based on RTP protocols / RTTP data transfer for VoIP telephony, and over the transport protocol UDP. Regarding 
delay, we note that the most "destructive" distortion imposed on the transmitted real-time signal (which is the VoIP telephony signal) is not so much the delay per-se, as the variation in this value (or jitter) [12]. In Figure 2, the maximum delay value reaches $200 \mathrm{~ms}$ (in the 18th line), and the jitter value is $76 \mathrm{~ms}$. Note that the packet loss level is not displayed by the route trace command.

Table 2. Tracing options.

\begin{tabular}{cr}
\hline Parameter & Value \\
\hline Maximum delay, ms & 200 \\
Jitter, ms & 76 \\
\hline
\end{tabular}

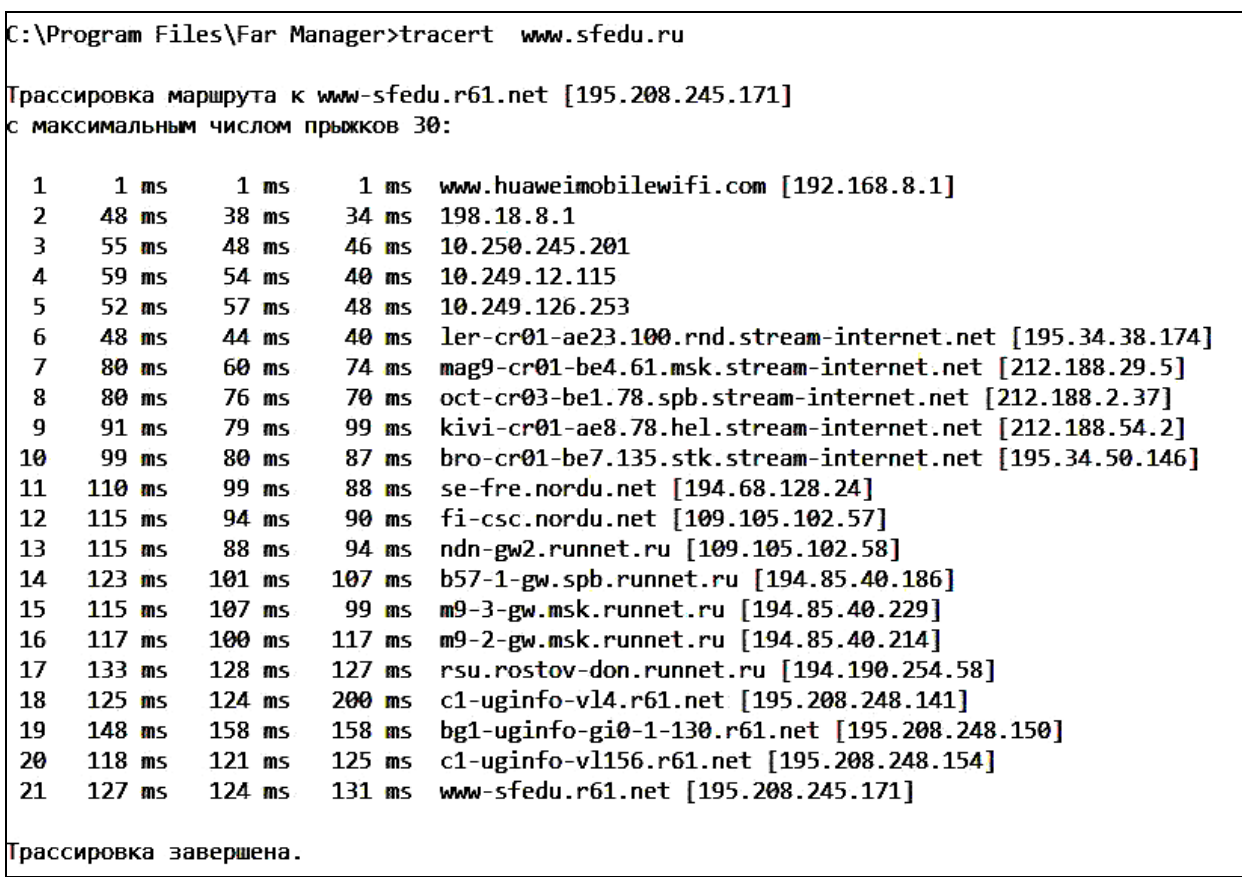

Figure 2. Example of an access route from an "external" VoIP device to a corporate network.

Case 2: a subscriber VoIP phone is connected to the corporate telecommunications network of the Southern Federal University (SFU) through the home Wi-Fi network. The VoIP subscriber is located in the city of Rostov-on-Don. The sequence of cities, given in Figure 3, is the same as that of the previous case: Rostov-on-Don - Moscow - St. Petersburg - Helsinki - Stockholm - Amsterdam - St. Petersburg - Moscow - Rostov-on-Don.

Table 3. Tracing options (second case).

\begin{tabular}{ccc}
\hline Trace number & 1 & 2 \\
\hline Maximum delay, ms & 208 & 113 \\
Jitter, ms & 65 & 2 \\
\hline
\end{tabular}

The parameters of these distortions of the data stream transmitted through the network can be used directly to estimate the quality of voice transmission, but such estimates are very approximate. However, these general distortions of the data stream are the primary sources of the secondary distortions which are directly perceived by VoIP subscribers - specific to speech transmission. Such distortions include, for example, delays in the arrival of a speech signal, intermittency and illegibility in speech, the presence of extraneous noise, the presence of an echo signal, and others which are considered in this paper. The evaluation of speech quality by measuring these secondary types of distortion is more accurate.

As we shall see, the jitter value found via the above analysis significantly exceeds the threshold level: i.e., that which is acceptable for high-quality speech transmission. Therefore, the problem of analyzing the quality of voice streams sent within the framework of connections with remote 
subscribers is of great importance for the purpose of further improving the parameters affecting the quality of voice transmission.

\begin{tabular}{|c|c|c|c|c|c|c|c|}
\hline \multicolumn{8}{|c|}{ c: \Users\Диимон〉tracert www.sfedu.ru } \\
\hline \multicolumn{8}{|c|}{$\begin{array}{l}\text { Tрассировка маршрута } \kappa \text { www-sfedu.r61.net [195.298.245.171] } \\
\text { С максимальным числом прыскков } 39 \text { : }\end{array}$} \\
\hline 1 & 2 & ms & 2 & ms & 1 & ms & myhome.ru [192.168.1.1] \\
\hline 2 & 3 & ms & 3 & ms & 3 & ms & 10.255 .61 .251 \\
\hline 3 & 5 & ms & 3 & ms & 6 & ms & 10.255 .61 .18 \\
\hline 4 & 5 & ms & 4 & ms & 3 & ms & 10.255 .61 .14 \\
\hline 5 & 4 & ms & 4 & ms & 3 & ms & ler-cre1-ae23.200.rnd.stream-internet.net [195.34.36.112] \\
\hline 6 & 25 & ms & 26 & ms & 25 & ms & mag9-cre1-be4.61.msk.stream-internet.net [212.188.29.5] \\
\hline 7 & 38 & ms & 37 & ms & 37 & ms & oct-cre3-be1.78.spb.stream-internet.net [212.188.2.37] \\
\hline 8 & 43 & ms & 45 & ms & 43 & ms & kivi-cre1-ae8.78.hel.stream-internet.net [212.188.54.2] \\
\hline 9 & 50 & ms & 49 & ms & 49 & ms & bro-cre1-be7.135.stk.stream-internet.net [195.34.58.146] \\
\hline 10 & 49 & ms & 51 & ms & 58 & ms & se-tug.nordu.net [194.68.123.24] \\
\hline 11 & 55 & ms & 56 & ms & 56 & ms & fi-csc.nordu.net [199.195.192.57] \\
\hline 12 & 81 & ms & 79 & ms & 79 & ms & ndn-gw2.runnet.ru [199.195.192.58] \\
\hline 13 & 143 & ms & 206 & ms & 298 & ms & 185.141.124.158. runnet.ru [185.141.124.15e] \\
\hline 14 & 85 & ms & 84 & ms & 84 & ms & m9-2-gw.msk. runnet.ru [194.85.48.53] \\
\hline 15 & 109 & ms & 199 & ms & 109 & ms & rsu.rostov-don.runnet.ru [194.199.254.58] \\
\hline 16 & 197 & ms & 197 & ms & 197 & ms & c1-uginfo-v14.r61.net [195.298.248.141] \\
\hline 17 & 184 & ms & 193 & ms & 163 & ms & bg1-uginfo-gie-1-139.r61.net [195.298.248.150] \\
\hline 18 & 123 & ms & 197 & ms & 107 & ms & c1-uginfo-v1156.r61.net [195.288.248.154] \\
\hline 19 & 111 & ms & 193 & ms & 184 & ms & WwW-sfedu.r61.net [195.208.245.171] \\
\hline \multicolumn{8}{|c|}{$\begin{array}{l}\text { c: \Users\Диимон>tracert www.sfedu.ru } \\
\text { Tрассировка маршрута к WWw-sfedu.r61.net [195.268.245.171] } \\
\text { c максимальным числом прысков } 38:\end{array}$} \\
\hline 1 & 6 & ms & & ms & 3 & ms & myhome.ru [192.168.1.1] \\
\hline 2 & 9 & ms & & ms & 4 & ms & 10.255 .61 .251 \\
\hline 3 & 4 & ms & 4 & ms & 5 & ms & 10.255 .61 .18 \\
\hline 4 & 4 & ms & 4 & ms & 4 & ms & 10.255 .61 .14 \\
\hline 5 & 5 & ms & 6 & ms & 7 & ms & ler-crø1-ae23.200.rnd.stream-internet.net [195.34.36.112] \\
\hline 6 & 27 & ms & 29 & ms & 27 & ms & mag9-cre1-be4.61.msk.stream-internet.net [212.188.29.5] \\
\hline 7 & 40 & ms & 46 & ms & 42 & ms & oct-cre3-be1.78.spb.stream-internet.net [212.188.2.37] \\
\hline 8 & 47 & ms & 45 & ms & 63 & ms & kivi-cre1-ae8.78.hel.stream-internet.net [212.188.54.2] \\
\hline 9 & 51 & ms & 52 & ms & 51 & ms & bro-cre1-be7.135.stk.stream-internet.net [195.34.50.146] \\
\hline 16 & 50 & ms & 50 & ms & 55 & ms & se-tug.nordu.net [194.68.123.24] \\
\hline 11 & 76 & ms & 75 & ms & 58 & ms & fi-csc.nordu.net [109.105.102.57] \\
\hline 12 & 80 & ms & 84 & ms & 82 & ms & ndn-gw2.runnet.ru [109.105.102.58] \\
\hline 13 & 92 & ms & 98 & ms & 91 & ms & 185.141 .124 .150 \\
\hline 14 & 91 & ms & 86 & ms & 85 & ms & m9-2-gw.msk.runnet.ru [194.85.46.53] \\
\hline 15 & 111 & ms & 111 & ms & 113 & ms & rsu.rostov-don.runnet.ru [194.190.254.58] \\
\hline 16 & 110 & ms & 111 & ms & 110 & ms & c1-uginfo-vl4.r61.net [195.208.248.141] \\
\hline 17 & 105 & ms & 105 & ms & 108 & ms & bg1-uginfo-gie-1-130.r61.net [195.208.248.150] \\
\hline 18 & 168 & ms & 110 & ms & 169 & ms & c1-uginfo-v1156.r61.net [195.268.248.154] \\
\hline 19 & 106 & ms & 105 & ms & 185 & ms & WWW-sfedu.r61.net [195.208.245.171] \\
\hline
\end{tabular}

Figure 3. Second example of an access route from an "external" VoIP device to a corporate network.

There are known methods for assessing the quality of voice transmission, based both on the analysis of primary distortions of the transmitted data stream, and on the analysis of secondary distortions characteristic of voice transmission specifically. This article discusses the causes and types of such distortions; compares the methods for assessing the presence of such distortions in order to choose the method most suitable for use in monitoring the quality of speech; and suggests methods for improving voice quality across corporate VoIP telephony systems by improving both the system VoIP-telephony, and its environment. The article ends with general conclusions concerning the results obtained.

One of the most promising methods for improving the quality of VoIP connections is associated with the use of more efficient codecs [13].

The use of the more modern Speex codec [14] allows for a significantly weakening of the requirements, i.e., the threshold values for the primary data transmission quality indicators in terms of what is needed in order to provide acceptable voice quality. Specifically, when using this codec, a satisfactory quality of voice transmission is ensured even with delays of up to $150 \mathrm{~ms}$, a jitter value of up to $15 \mathrm{~ms}$, and losses of up to $10 \%$ of packets. Thus, in comparison with the recommendations of ITU-T G.712 [15], the threshold value for the permissible variation in delays is increased by a factor of 1.5, and the percentage of admissible data loss is increased tenfold. [2, 9]. 
Note, however, that the Speex codec is not the best currently available. The electronic resource devoted to this codec [14] provides the information that the Speex codec is surpassed, according to all indicators, by the new freely distributed Opus codec [16]. Note also that the Opus codec, developed in 2011 (the latest version of this codec was released in July 2016), has already been standardized by the IETF (Internet Engineering Task Force) as standard RFC 6716 [17]. This standard combines the technologies of such well-known codecs as Skype SILK [18] and Xipn.Org CELT [19]. Because of the noted advantages of the codec, Opus decided to implement it in the VoIP-PBX IP4Tel system, which is the basis of the system, proposed here, for monitoring the quality of voice transmission across corporate VoIP-telephony systems.

\section{Conclusions and future work}

For this study, a comparative analysis and a development of the methods for assessing the quality of voice transmission across VoIP telephony networks was carried out. Also, a comparative analysis of the following VoIP codecs was performed: Speex, Opus, Skype SILK and Xipn.Org CELT. This was a comparison by the following criteria: allowable delay, allowable losses and allowable jitter (delay variation). This comparison showed that the Opus codec is the best choice at present. Thus, the Opus codec was chosen for the further development of the VoIP-telephony system of the Southern Federal University.

We believe that once our program of implementation of the methods proposed in this work has been completed, the results will be of considerable interest to many organizations using corporate VoIP telephony systems that allow remote access of subscribers through networks of third-party telecommunications operators.

\section{References}

[1] Sukhov A M, Sultanov T G and Polukarov D Y 2012 The method of available bandwidth estimation of IP-connection based on variable packet size Elektrosvyaz 11 39-42

[2] Bukatov A A, Zaitsev N D and Berezovsky A N 2017 Methods for monitoring and improving the quality of voice transmission for the system of corporate VoIP telephony SFU Modern information technologies: trends and development prospects. Materials of the XXIV scientific conference 47-52

[3] Calyam P, Ekici E, Lee C G, Haffner M and Howes N 2007 A "GAP-Model" based framework for online VVoIP QoE measurement Journal of Communications and Networks 9(4) 446-456

[4] G.107: E-model: a computational model for use in transmission planning (Access mode: http://www.itu.int/rec/T-REC-G.107/) (2017-21-04)

[5] P.861: Objective quality measurement of telephone-band (300-3400 Hz) speech codecs (Access mode: https://www.itu.int/rec/T-REC-P.861/en/) (2017-21-04)

[6] P.862: Perceptual evaluation of speech quality (PESQ): An objective method for end-to-end speech quality assessment of narrow-band telephone networks and speech codecs (Access mode: http://www.itu.int/rec/T-REC-P.862/en/) (2017-21-04)

[7] P.563: Single-ended method for objective speech quality assessment in narrow-band telephony applications (Access mode: http://www.itu.int/rec/T-REC-P.563-200405-I/) (2017-21-04)

[8] Kurittu A 2006 Validation of ITU-T P. 563 single-ended objective speech quality measurement Journal of the Audio Engineering Society 54(11) 1092-1098

[9] Lukinskikh K S 2015 Unbiased Assessment Method For Voice Communication in Cloud VoIPTelephony Journal of Theoretical and Applied Information Technology 80(1) 75

[10] Gugel Yu V, Izhvanov Yu L, Kurakin D V 2010 Development of international connectivity of the federal computer network RUNNet Proceedings of the XVII All-Russian Scientific and Methodical Conference "Telematics'2010" 273-275

[11] Bolot J C 1993 Characterizing end-to-end packet delay and loss in the internet Journal of High Speed Networks 2(3) 305-323

[12] Karam M J and Tobagi F A 2001 Analysis of the delay and jitter of voice traffic over the internet Twentieth annual joint conference of the IEEE Computer and Communications Societies 2 824-833 
[13] Singh H P, Singh S and Singh J 2011 Real time analysis of VoIP system under pervasive environment through spectral parameters International Journal of Computer Applications 31(2) $1-8$

[14] Speex: A Free Codec For Free Speech (Access mode: https://speex.org/) (2017-21-04)

[15] G.712: Transmission performance characteristics of pulse code modulation channels (Access mode: https://www.itu.int/rec/T-REC-G.712-199209-S/en/) (2017-21-04)

[16] Opus Codec (Access mode: http://opus-codec.org/) (2017-21-04)

[17] RFC 6716 - Definition of the Opus Audio Codec (Access mode: https://tools. ietf.org/html/rfc6716/) (2017-21-04)

[18] SILK - Wikipedia (Access mode: https://en.wikipedia.org/wiki/SILK/) (2017-21-04)

[19] CELT: new audio codec from xipn.org (Access mode: https://www.linux.org.ru/ news/ opensource/2601080/) (2017-21-04)

\section{Acknowledgements}

This work falls within the public tasks allotted to the Ministry of Education and Science of the Russian Federation (2.974.2017/4.6) and was carried out with the support of grant RFBR 16-07-00218a. 